\title{
Solar Cycle Variation in Geomagnetic External Spherical Harmonic Coefficients
}

\author{
Leroy R. Alldredge, ${ }^{*}$ Charles O. Stearns, ${ }^{*}$ \\ and Masahisa Sugiura** \\ *U.S. Geological Survey, Denver, CO 80225, U.S.A. \\ **Laboratory for Planetary Atmospheres, NASA/Goddard Space \\ Flight Center, Greenbelt, MD 20771, U.S.A.
}

(Received April 11, 1978; Revised January 18, 1979)

The first degree external spherical harmonic coefficients are obtained for each year of a 12-year period centered at 1958.5, using annual mean values of $X, Y$, and $Z$ components from 54 magnetic observatories. Values of the coefficient $g_{1 e}^{0}$ of the zonal first degree harmonic clearly show a solar cycle variation. The peak-to-peak amplitude of the variation is approximately 29 $\mathrm{nT}$, which is consistent with values obtained earlier by first filtering data to retain only those variations having periods near the solar cycle and then subjecting the filtered data to a spherical harmonic analysis. The variation in $g_{1 e}^{0}$ is found to correlate extremely well with the annual mean $D_{\mathrm{st}}$ index and with the annual number of days having $A p \geq 60$. Based on statistics of the mean square successive difference, an explanation is presented why the obtained solar cycle variation in $g_{1 e}^{0}$, which is very much smaller in magnitude than the standard deviations calculated by the conventional method, is statistically meaningful. The determined absolute (not relative) values of $g_{1 e}^{0}$ are in agreement, within several nanotesla, with the expectation from a theoretical model of solar wind compression of the magnetosphere and an analysis of the $D_{\text {st }}$ index.

\section{Introduction}

GAUSs' (1838) spherical harmonic analysis of the geomagnetic field was the first application of his potential theory to actual observational data. From this analysis Gauss concluded that to the accuracy of the data available, the cause of the geomagnetic field was internal to the earth. With improved magnetic observations made since Gauss' analysis the spherical harmonic coefficients for the external part of the geomagnetic potential have been obtained by many workers. Some of these results have been summarized by Alldredge and Stearns (1974). The scatter of the values of these external coefficients is so great that one must conclude that the results reflect considerable amount of 
noise in the data or inadequacies in the methods of analysis used.

On the other hand, Yukutake (1965), Alldredge (1976), Riven (1976), Courtillot and Le Mouel (1976), and Harwood and Malin (1977) have found that values of the first degree external coefficients vary consistently with the solar cycle. In all of these analyses, when the solar cycle effect was being investigated, the data were first filtered to retain only those variations with periods near the solar cycle. The external coefficient, $g_{1 e}^{0}$, and the internal coefficient, $g_{1 i}^{0}$, were found to be well correlated with the sunspot number. The ratio of these coefficients was useful in discussing upper mantle conductivity. These results suggest that the external coefficients thus obtained have a real meaning.

This success with the solar cycle variation in the external coefficients encouraged us to attempt to determine the external first degree coefficients by a direct application of spherical harmonic analysis to the unfiltered observatory data. It was thought that results of an analysis of a homogeneous data set, i.e., data from the same observatories for each year of a specified period, would show a true solar cycle modulation of the external field if not its absolute values. Such an analysis would test the feasibility of routinely including low degree external spherical harmonics in deriving geomagnetic field models.

It was not expected that the solar cycle effect on the internal coefficient $g_{1 i}^{0}$ obtained from the unfiltered obervatory data would be obvious at all because of the very large contribution to $g_{1 i}^{0}$ from internal core sources. To get the solar cycle variation in $g_{1 i}^{0}$ would require the use of filtering techniques which were being avoided in this paper. Because of this, arguments about the ratio of $g_{1 i}^{0} / g_{1 e}^{0}$ and the resulting upper mantle conductivity are not discussed in this paper. This topic has been treated in several of the references cited earlier. It is also being treated by YUKUTAKE and CAIN (1979) in a forthcoming study which was done partly in collabration with this study.

\section{Computation of External Coefficients}

The usual expression for the geomagnetic potential is

$$
\begin{aligned}
V= & a \sum_{n=1}^{\infty} \sum_{m=0}^{n} P_{n}^{m}(\cos \theta)\left\{(a / r)^{n+1}\left(g_{n i}^{m} \cos m \lambda+h_{n i}^{m} \sin m \lambda\right)\right. \\
& \left.+(r / a)^{n}\left(g_{n e}^{m} \cos \mathrm{m} \lambda+h_{n e}^{m} \sin m \lambda\right)\right\},
\end{aligned}
$$

where $a$ is the earth's radius, $P_{n}^{m}$ are associated Legendre polynomials, $(r, \theta, \lambda)$ are radial distance, colatitude, and longitude, and $g$ 's and $h$ 's are the coefficients. The north, east, and vertical components of the magnetic field are given by $X=r^{-1} \partial V / \partial \theta, Y=-(r \sin \theta)^{-1} \partial V / \partial \lambda$, and $Z=\partial V / \partial r$, respectively (e.g., Vestine et al., 1947). If all measurements of $X, Y$, and $Z$ are made on the surface of a 
spherical earth, an analysis of $X$ and $Y$ combined yields the sums of the external and internal coefficients, $g_{n e}^{m}+g_{n i}^{m}$, and an analysis of $Z$, the differences, $n g_{n e}^{m}-(n+1) g_{n i}^{m}$. From these two sets the external and internal coefficients, $g_{n e}^{m}$ and $g_{n i}^{m}$, can be separated. This procedure was used by most investigators before large scale computers became readily available.

In principle, of course, both the external and internal coefficients can be evaluated in one step by the method of least squares. This procedure was used in this investigation. It was further assumed that only the first degree external coefficients are nonzero. This assumption adds only the three unknowns, $g_{1 e}^{0}$, $g_{1 e}^{1}$, and $h_{1 e}^{1}$, to the usual set of internal coefficients. The earth is assumed to be spherical which should not result in errors in the first order external coefficients because the field represented by these coefficients is essentially independent of $r$.

It was desired to have a sufficiently long series of annual means of $X, Y$, and $Z$ in order to see a solar cycle modulation. Five year averages used by MALIN (1969), are inadequate for the present analysis because of oversmoothing. Also, identical observatories must be used for each year so that the results are not influenced by changes in observatories. To optimize chances of success it was decided to narrow the attempt to the 12-year period from 1953 to 1964 which covers the most active solar cycle in the past 200 years on the basis of the sunspot number. Although twelve years is short, the selected period provides a maximum number of observatories with continuous records.

All annual means of $X, Y$, and $Z$ components available at the World Data Center A were examined for completeness and were screened to avoid data affected by noise. Data from a few observatories in or near the equatorial and auroral electrojet regions were rejected because of the presence of noisy annual means caused either by bad baseline control or erratic jet sources. Fifty-four observatories thus selected are listed in Table 1. Their distribution is shown in Fig. 1. The observatory locations and the component data are available at the World Data Center A; see also World Data Center A (1974) in the References for the observatory locations. The missing component values for 1953 for Simosato were extrapolated. The discontinuity in the Honolulu data caused by a change in location in 1961 was removed by normalizing the data taken after the move to those obtained at the earlier location. The Fredericksburg data set was similarly continued back to 1953 by using the Cheltenham data and applying proper adjustments to the baselines. Also values for Pilar for the years 1957, 1958 , and 1959 were interpolated.

Figure 2 shows plots of the three first degree external coefficients derived by a spherical harmonic analysis in which internal coefficients through degree and order $6\left(N_{\max }=6\right)$ and external coefficients for degree 1 were determined. Of the 54 observatories used, 25 are in the European area. Therefore a weight 
Table 1. List of observatories

\begin{tabular}{|c|c|}
\hline Amberley & Nurmijarvi* \\
\hline Apia & Panagyurishte* \\
\hline Binza & Pilar \\
\hline Chambon la Foret* & Quetta \\
\hline Coimbra* & Rude Skov* \\
\hline Dikson & San Juan \\
\hline Dumbas* & San Miguel \\
\hline Dusheti & Simosato \\
\hline Ebro* & Sitka \\
\hline Eskdalemuir* & Sodankyla* \\
\hline Fredericksburg & Stepanovka* \\
\hline Fürstenfeldbruck* & Swider* \\
\hline Godhavn & Tamanrasset \\
\hline Hermanus & Tananarive \\
\hline Honolulu & Toledo* \\
\hline Hurbanovo* & Toolangi \\
\hline Istanbul* & Tromso* \\
\hline Kakioka & Tucson \\
\hline Krasnaya Pakhra* & Valentia* \\
\hline Lerwick* & Vassouras \\
\hline Lovo* & Voyeykovo* \\
\hline M'Bour & Vysokay Dubrava \\
\hline Macquarie Island & Wingst* \\
\hline Manhay* & Witteveen* \\
\hline Memambetsu & Yakutsk \\
\hline Muntinlupa & Yuzhno Sakhalsk \\
\hline Niemegk* & Zaymishche \\
\hline
\end{tabular}

* indicates that the observatory is in the European Area.

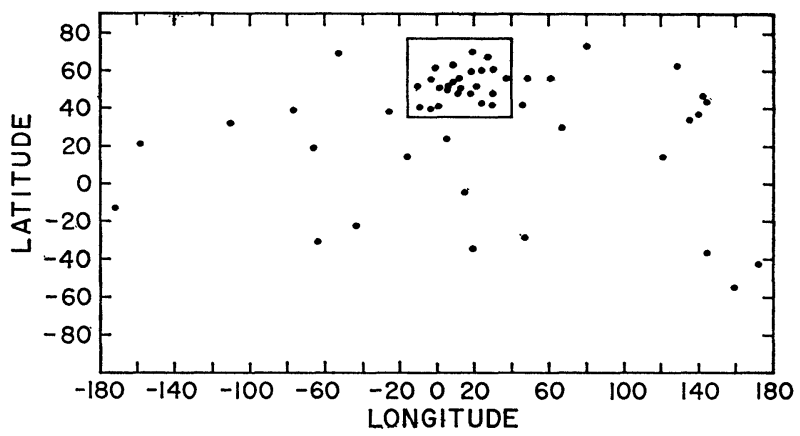

Fig. 1. Distribution of observatories used in this study. The $25 \mathrm{ob}-$ servatories in the European Area, shown with an * in Table 1, are included in the inset box. 


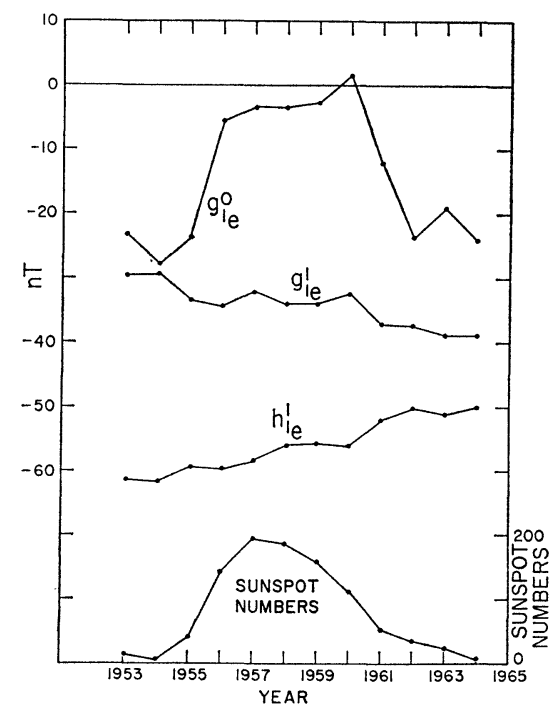

Fig. 2. First degree external coefficients, determined together with internal coefficients up to $N_{\max }=6$. A weight of 0.2 was assigned to observatories in the Europian Area (see Table 1). The annual average sunspot numbers are also shown.

of 0.2 was assigned to each of the 25 European observatories, which are marked with asterisks in Table 1.

\section{Discussion}

\subsection{Number coefficients determined}

There is no unambiguous way to determine exactly how many coefficients should be taken in a spherical harmonic field model for a given set of data. The distribution of the observatories is often so poor that the advantages of applying fine theoretical techniques as discussed by Fougere (1963) are lost. It is interesting to note that when Fougere (1965) applied his method of orthogonal functions to data from 80 observatories, he determined in the end 77 coefficients which included every coefficient that goes with $N_{\max }=8$ except the last three. That is, the application of his method did not eliminate any of the lower order terms, and the number of coefficients matched quite well what others had used previously. When the coefficients in a geomagnetic spherical harmonic analysis are greatly overdetermined, the RMS residual for all three components is normally about $200 \mathrm{nT}$ (Peddie and Fabiano, 1976), which is attributed mainly 
Table 2. Average and range of standard deviations, and Residuals for 12 years, based on annual means, with weight $=0.2$ for European observatories.

\begin{tabular}{|c|c|c|c|c|}
\hline & \multicolumn{3}{|c|}{ Standard deviations of coefficients (nT) } & \multirow{2}{*}{$\frac{\text { RMS }}{\text { Residuals (nT) }}$} \\
\hline & $g_{1 e}^{0}$ & $g_{1 e}^{1}$ & $h_{1 e}^{1}$ & \\
\hline Min. & 102.7 & 105.8 & 141.1 & 154.0 \\
\hline Ave. & 103.4 & 106.7 & 142.2 & 155.3 \\
\hline Max. & 104.5 & 107.7 & 143.5 & 156.7 \\
\hline
\end{tabular}

to local anomalies. Of course, when the number of unknowns equals the number of observations the RMS residual becomes zero. MALIN (1969) in working with a data set from 80 observatories chose $N_{\max }=6$ because: 1 ) he considered that 48 spherical harmonic coefficients was a reasonable number to deduce from data obtained at 80 positions; 2) a very good fit to the data was obtained by a sixth degree analysis and the fit was not greatly improved even by a ninth degree analysis using twice as many coefficients.

With the above arguments in mind, solutions were obtained for several values of $N_{\max }$. The results indicated that degree 6 gave a good representative solution. Table 2 shows that with $N_{\max }=6$ the RMS residual was approximately $155 \mathrm{nT}$. With $N_{\max }=4$ the RMS residual was about $700 \mathrm{nT}$. These values bracket the RMS expected from local anomalies. Thus the selection of $N_{\max }=6$ appears to be very reasonable.

\subsection{Solar cycle effect}

In Fig. 2 a solar cycle effect is evident in $g_{1 e}^{0}$, but no such distinctive variations are seen in $g_{1 e}^{1}$ and $h_{1 e}^{1}$. These three coefficients represent a tilted uniform field from external sources; $\theta_{0}$ and $\lambda_{0}$ in the ratios $g_{1 e}^{1} / g_{1 e}^{0}=\tan \theta_{0} \cos \lambda_{0}$ and $\lambda_{1 e}^{1} / g_{1 e}^{0}=$ $\tan \theta_{0} \sin \lambda_{0}$, give the geographic colatitude and longitude of the north pole of the axis parallel to this tilted uniform external field. If one assumes that the field represented by these external coefficients is caused by an equatorial ring current lying in a plane perpendicular to the axis of the dipole field, then it is clear that the magnitudes of the above ratios would equal those of the corresponding ratios for the internal coefficients of the main field which for the International Geomagnetic Reference Field (ZMUDA, 1971) are 0.7 and -0.19, respectively. The small values of these ratios would make it difficult to see the solar cycle effects in $g_{1 e}^{1}$ and $h_{1 e}^{1}$. The peak-to-peak variation of $g_{1 e}^{0}$, as determined from Fig. 2, is approximately $29 \mathrm{nT}$. This is just a little larger than that obtained by ALLDREDGE (1976) for earlier, less pronounced sunspot cycles using an entirely different method.

\subsection{Relative accuracy of the coefficient absolute values, and their time-variation}

Although the variation with time of these external coefficients agrees with 
the expected behavior, there is no good way to assess the quality of the absolute values that have been derived. Even if the data are more extensive, there is no guarantee that the absolute values are good. Standard deviations of the coefficients were determined using the commonly used formula (e.g., BROWNLEE, 1965). The minimum, maximum, and average of these standard deviations for the 12 years are given in Table 2 . These standard deviations are large compared to the solar cycle variation discussed above. It is shown below that although the absolute values may not be accurately determined, the homogeneous data set has a consistency that permits a much smaller uncertainty in determining the variation from one year to the next.

The fact that the year-to-year consistency was much better than that implied by the large standard deviations shown in Table 2 was proved as follows. Differences of annual means of $X, Y$, and $Z$ were taken for successive years for each observatory. These differences were then used to determine year-to-year differences in the harmonic coefficients by the same method as that used in the original analysis. When the year-to-year differences of the coefficients were successively added from 1954 through 1964, the resultant series was identical, to the second decimal, to the series of $g_{1 e}^{0}$ obtained above by using the original $(X, Y, Z)$ data set except that the new series refers to the level at 1953. This is, however, expected because the least squares procedure is a linear process. It is noted here that the agreement of the two sets of results was also found in all the internal coefficients. The standard deviations calculated for the year-toyear difference scheme were on the average only about one fiftieth of the corresponding standard deviations associated with the original coefficients. The averages and ranges of the standard deviations for the year-to-year differences are shown in Table 3. Detailed discussions on these standard deviations are given below.

\subsection{Correlation with Ap index}

T. Yukutake called attention to a very close correlation between the external zonal first degree spherical harmonic coefficients and the annual number of days having the activity index $A p$ equal to or greater than a given number as noted in Fig. 3. The $A p$ index is the mean of the eight $A p$-values for a day, and the $A p$-values are obtained from the planetary quasi-logarithmic index $K p$. The correlation between the first order zonal harmonic coefficient and the annual number of a days having $A p \geq 60$ is extremely high.

\subsection{Correlation with $D_{\text {st }}$ index}

Another very striking correlation is found between the $g_{1 e}^{0}$ variation (curve b in Fig. 3) and the variation in the annual mean equatorial $D_{\mathrm{st}}$ index (curve c 


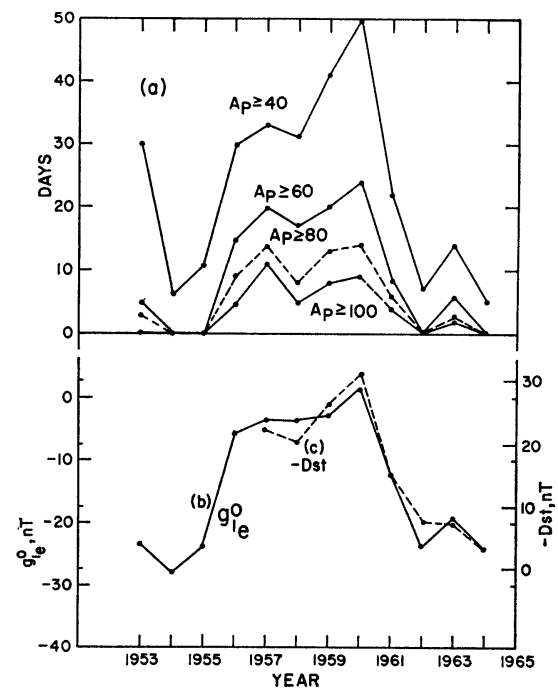

Fig. 3. (a) annual number of days having $A p \geq$ $40,60,80$, and 100 , (b) first degree zonal external coefficients $g_{1 e}^{0}$ (same as in Fig. 2), and (c) annual average $D_{\text {st }}$ with reversed sign.

in Fig. 3). The annual averages of the $D_{s t}$ index are based on an updated, revised version of the data given by Sugiura and Poros (1971). In Fig. 3, $D_{\text {st }}$ is plotted with reversed sign because with increasing solar activity the equatorial geomagnetic field is decreased and by definition $D_{\mathrm{st}}$ is more negative. Values of $-D_{\mathrm{st}}$ are plotted with an offset of $-27 \mathrm{nT}$ to make the average of $-D_{\text {st }}$ equal to that of $g_{1 e}^{0}$ for the years 1957 to 1964 for which $D_{\text {st }}$ is plotted. The correlation coefficient between $g_{1 e}^{0}$ and $-D_{\mathrm{st}}$ for these years is 0.97 . Based on the standard $t$-test the probability that this correlation should arise by random sampling from an uncorrelated population is less than 0.01 . Therefore the correlation is highly significant statistically.

\subsection{Solar wind effects}

The obtained variation in $g_{1 e}^{0}$ should include, besides the effect of the solar modulated ring current, all other external magnetic sources that may or may not vary with the solar cycle. One would expect very crudely that the part of $g_{1 e}^{0}$ caused by the ring current would be weakest (i.e., $g_{1 e}^{0}$ nearest zero) in the sunspot minimum years and strongest (i.e., $g_{1 e}^{0}$ furthest from zero) in the years of high sunspot number. The $D_{\text {st }}$ plot shows this trend, while $g_{1 e}^{0}$ deviates more from zero in the years of low solar activity. Let us assume for the moment that the offset caused by sources other than the ring current is $-27 \mathrm{nT}$ and ask what 
could produce such an effect. According to MEAD's (1964) model for the magnetospheric boundary, the effect of the compression of the geomagnetic field by a steady solar wind that places the subsolar magnetopause at $r_{b}$ earth radii can be represented by $g_{1 e}^{0}=-0.2515 \times 10^{+5} / r_{b}^{3}$ (in $\mathrm{nT}$ ). If the magnetopause distance is assumed to be constant throughout the period considered here, the offset of $g_{1 e}^{0}$ in Fig. 3 of approximately $-27 \mathrm{nT}$ would correspond to the magnetopause distance of 9.8 earth radii. If one assumes the presence of such a steady solar wind, the observed solar cycle variation in $g_{1 e}^{0}$ can be interpreted as being mainly caused by a solar cycle variation in the ring current, ignoring other, presumably smaller, effects. For the sake of clarity of argument these other effects are completely ignored in the following discussion. Then the peak-to-peak amplitude of the solar cycle variation in the ring current during the period 1953 to 1964 was $29 \mathrm{nT}$.

However, the solar wind pressure may not be constant throughout the solar cycle. As a matter of fact, purely logically speaking, an explanation opposite to the one presented above can be put forward. That is, one may argue that the ring current remained the same during the solar cycle and that a variation in the solar wind pressure was solely responsible for the observed change in the $g_{1 e}^{0}$. This explanation, however, becomes absurd because near the solar maximum years the magnetopause distance must go to infinity, i.e., the solar wind pressure must be near zero; or at least qualitatively, the solar wind pressure must be weakest when solar activity is highest, which is unlikely. A more reasonable interpretation would be to consider the solar cycle variation in $g_{1 e}^{0}$ to be mainly from that in the ring current, possibly with a secondary contribution, among other effects, from a solar cycle variation in the solar wind pressure. If the solar wind pressure increases with increasing solar activity, the $g_{1 e}^{0}$ variation from the ring current obtained under the assumption of a constant solar wind would be an underestimate.

\subsection{The subsolar magnetopause location}

Having presented a crude argument, we now examine the problem in a little more detail. The average position of the subsolar magnetopause as observed by the $\operatorname{Imp} 1,2$, and 3 satellites during the time intervals November 1963 to May 1964 (Imp 1) October 1964 to April 1965 (Imp 2), and May 1965 to May 1967 (Imp 3) was approximately 10.5 earth radii (FAIRFIELD, 1968). In a later paper FAIRFIELD (1971) placed the average subsolar magnetopause at 10.8 to 11.0 earth radii (depending upon geometric refinements in the modeling) based on an expanded data set drawn from the observations by Imp's 1, 2, 3, and 4 and Explorers 33 and 35. From an analysis of magnetometer data obtained by Explorer 12 in the period August to November 1961 Cahill and Patel (1967) 
found the average magnetopause position near the noon meridian to be 10.7 earth radii. Based on these observations the average subsolar magnetopause location in the period 1963-1964 was probably in the vicinity of 10.5 to 11.0 earth radii. These values corrspond to -22 to $-19 \mathrm{nT}$ in terms of the coefficient $g_{1 e}^{0}$ if Mead's formula quoted above is used. These values agree with those of $g_{1 e}^{0}$ for the 1963-1964 period in Fig. 3 within a few nanotesla. Nevertheless on the basis of the standard deviations associated with the determined $g_{1 e}^{0}$ as given in Table 4, this agreement must be disregarded as being not statistically significant.

\subsection{Further examination of the statistics of coefficient determination}

There is an apparent paradox in this discussion. The plots of $g_{1 e}^{0}$ in Fig. 3 were obtained by a least squares fitting for each year independently of other years. As described earlier the $g_{1 e}^{0}$ sequence can also be obtained by successively adding year-to-year differences to the absolute level of $g_{1 e}^{0}$ for the base year, 1953. The standard deviations ( $\sigma$ 's) associated with the differences $\Delta g_{1 e}^{0}$ were found to be quite small as was shown in Table 3. Also the correlation between $g_{1 e}^{0}$ and $D_{\text {st }}$ was found to be extremely high. On the other hand, the $\sigma$ 's for $g_{1 e}^{0}$ when determined for individual years were greater than $100 \mathrm{nT}$ as shown in Table 4.

Table 3. Average and range of sandard deviations (nT) for 12 years, based on year-toyear successive differences of annual means, with the same weight as in Table 2.

\begin{tabular}{lccc}
\hline & $\Delta g_{1 e}^{0}$ & $\Delta g_{1 e}^{1}$ & $\Delta h_{1 e}^{1}$ \\
\hline Min. & 1.9 & 1.9 & 2.5 \\
Ave. & 2.4 & 2.4 & 3.2 \\
Max. & 3.5 & 3.6 & 4.8 \\
\hline
\end{tabular}

Table 4. Standard deviation ( $\sigma$ 's) for $g_{1 e}^{0}$ for individual years.

\begin{tabular}{cc}
\hline Year & $\sigma$ in nT \\
\hline 1953 & 102.69 \\
1954 & 102.92 \\
1955 & 102.81 \\
1956 & 102.89 \\
1957 & 103.10 \\
1958 & 103.63 \\
1959 & 103.29 \\
1960 & 103.84 \\
1961 & 104.18 \\
1962 & 104.30 \\
1963 & 104.25 \\
1964 & 104.47
\end{tabular}


These two seemingly paradoxical aspects of the same results (Fig. 3) can be interpreted in the following way. Although the (absolute) $g_{1 e}^{0}$ coefficients were determined for $N$ individual years separately, the $(X, Y, Z)$ data sets for these $N$ years are so well correlated with each other that the $N$ standard deviations themselves are not really independent. That this is really so can be shown by the mean square successive difference test as follows. Let us denote the $g_{1 e}^{0}$ sequence by $X_{1}, X_{2}, \ldots, X_{N}$, and assume a null hypothesis that this sequence consists of independent observations from a normally distributed population. If we define $d^{2}$ from the successive differences by

$$
d^{2}=\frac{1}{N-1} \sum_{i=1}^{N-1}\left(X_{i+1}-X_{i}\right)^{2}
$$

the expected value of $d^{2} / 2, E\left(d^{2} / 2\right)$, is equal to the variance, $\sigma^{2}$, of the population. If we denote an estimate of $\sigma^{2}$ by $s^{2}$, it is known that the expected value and the variance of $\left(d^{2} / 2\right) / s^{2}$ are given by

$$
E\left[\frac{d^{2} / 2}{s^{2}}\right]=1
$$

and

$$
V\left[\frac{\left(d^{2} / 2\right)}{s^{2}}\right]=(N-2) /\left(N^{2}-1\right)
$$

respectively and thus that the test statistic

$$
u_{p}=\left(d^{2} / 2-1\right) /\left\{(N-2) /\left(N^{2}-1\right)\right\}
$$

is distributed approximately as a standardized normal deviate (vON NeUMANN et al., 1941; HART, 1942).

The underlying idea of the test is that if the consecutive observations are correlated with their predecessors, the successive differences tend to be smaller than they would be under complete randomness and that therefore the expected value of $d^{2} / 2$ is less than $s^{2}$. In the present case, $d^{2} / 2=65$, while $s^{2}=114$, and so the sequence $X_{i}$ is correlated. Now the test statistic defined by Eq. (4) is $u_{p}=-2.64$; hence using a table of the cumulative standardized normal distribution function, the probability $P$ for this value is 0.004 . Thus we can reject the null hypothesis of randomness with a risk of less than $1 \%$.

Thus, so far as the variation in $g_{1 e}^{0}$ is concerned, the eleven standard deviations for $\Delta g_{1 e}^{0}$, are the relevant quantities for the solar cycle variation, and not the twelve standard deviations (in Table 4) for $g_{1 e}^{0}$ themselves. However, for the absolute value of the reference level for the variation, the standard deviation for some base year must be regarded as a relevant statistical quantity. The standard deviations for $g_{1 e}^{0}$ are between $102.6 \mathrm{nT}$ and $104.5 \mathrm{nT}$ (Table 4). Taking 
1964 as the base year, $\sigma$ is $104 \mathrm{nT}$. Let us now examine what this $\sigma$ value might mean. To do so we recapitulate the question of the meaning of $g_{1 e}^{0}$. We begin with the $D_{\text {st }}$ index. Even on magnetically quiet days the equatorial magnetic field is decreased by the equatorial ring current in the magnetosphere (SUGIURA, 1973). The $D_{s t}$ index is defined such that the average $D_{\text {st }}$ for quiet days is zero. In 1964 the annual average $D_{\text {st }}$ was $-3 \mathrm{nT}$, meaning that the annual average of the horizontal component, $H$, at the equator on the earth's surface was $3 \mathrm{nT}$ below that for the quiet days. If we assume that in 1964 the annual average position of the subsolar point on the magnetopause was at $10.75( \pm 0.25)$ earth radii from the earth's center, the contribution to $g_{1 e}^{0}$ from the solar wind compression of the magnetosphere is $-20( \pm 2) \mathrm{nT}$, based on Mead's model. If we further assume that the geocentric distance of the subsolar magnetopause on the quiet days did not appreciably differ from that on the annual average, then taking $3 \mathrm{nT}$ as the difference between the annual average and the quiet-day average, the expected value of $g_{1 e}^{0}$ for 1964 is $-17( \pm 2) \mathrm{nT}$; here it should be remembered that a positive $g_{1 e}^{0}$ corresponds to a southward magnetic field. The value of $g_{1 e}^{0}$ determined for 1964 by the method of least squares was $-24 \mathrm{nT}$. Therefore there is a discrepancy of $7 \mathrm{nT}$ between the expected value and the determined value of $g_{1 e}^{0}$. This difference is very much smaller than the value of $\sigma$, i.e., 104 $\mathrm{nT}$. The question then is: Is the difference of $7 \mathrm{nT}$ significant? If we take the $\sigma$ calculated by the conventional method literally, such a small difference is certainly not significant. Nevertheless, on the basis of other physical reasoning we believe that the small difference of $7 \mathrm{nT}$ cannot be discarded as being a mere number that is statistically insignificant. The large $\sigma$ of $104 \mathrm{nT}$ is intrinsically associated with the method of analysis, namely the spherical harmonic analysis using a finite number of terms and observed field values that are at least a thousand times greater than the field caused by the external sources, rather than with the true statistical scatter in $g_{1 e}^{0}$. This question concerns the theoretical basis of the statistic involved in the least squares determination used here, and is outside the scope of this paper. We merely point out the apparent arbitrariness in the distributions of the spherical harmonic coefficients determined by the method of least squares. As one increases the number of terms, $n$, the value of $\sigma$ decreases and in general, beyond some number $n_{1}$, the improvement in the fit becomes questionable. However, when $n$ equals the number of known variables, the mathematical determination of the coefficients becomes exact. This certainly does not mean that the fit is perfect physically.

\section{Conclusions}

We have shown that the solar cycle variation in the coefficient $g_{1 e}^{0}$ deter- 
mined by a spherical harmonic analysis using the external coefficients of degree $1\left(g_{1 e}^{0}, g_{1 e}^{1}\right.$, and $\left.h_{1 e}^{1}\right)$ is highly correlated with the variation in the average $D_{\mathrm{st}}$. That this conclusion is statistically meaningful is shown by use of successive (yearto-year) differences in $g_{1 e}^{0}$ and by a statistical test of mean square successive difference.

The determined $g_{1 e}^{0}$ agrees within several nanotesla with the expectation based on our present knowledge of the solar wind and ring current effects.

The authors were greatly helped by discussions with T. Yukutake, J.C. Cain, and D.H. Fairfield.

\section{REFERENCES}

AlldRedGe, L.R., Effects of solar activity on annual means of geomagnetic components, $J$. Geophys. Res., 81, 2990-2996, 1976.

Alldredge, L.R. and C.O. Stearns, A discussion of sources and description of the earth's magnetic field and its secular variations, J. Geomag. Geoelectr., 26, 393-404, 1974.

Brownlee, K.A., Statistical Theory and Methodology in Science and Engineering, second edition, 590 pp., John Wiley and Sons, Inc., New York, 1965.

Cahill, L.H., Jr. and V.L. Patel, The boundary of the geomagnetic field, August to November, 1961, Plan. Space Sci., 15, 997-1033, 1967.

Courtillot, V. and J.L. Le Mouel, On the long period variations of the earth's magnetic field from 2 months to 20 years, J. Geophys. Res., 81, 2941-2950, 1976.

FAIRFIELD, D.H., Average magnetic field configuration of the outer magnetosphere, J. Geophys. Res., 73, 7329-7338, 1968.

FAirfield, D.H., Average and unusual locations of the earth's magnetopause and bow shock, J. Geophys. Res., 76, 6700-6716, 1971.

Fougere, P.F., Spherical harmonic analysis-A new method and its verification, J. Geophys. Res., 68, 1131-1139, 1963.

Fougere, P.F., Spherical harmonic analysis - A new model derived from magnetic observatory data for epoch 1960.0, J. Geophys. Res., 70, 2171-2179, 1965.

GaUss, C.F., Allgemeine theorie des Erdmagnetismus, in Resultate aus den Beobachtungen des Magnetischen Verin im Jahie, 1838 (reprinted in Werke, band 5, 121-193, Koniglichen Gessellschaft der Wissenschaften, Gottingen, 1877), 1839.

Hart, B.I., Significance levels for the ratio of the mean square successive difference to the variance, Ann. Math. Stat., 13, 445-447, 1942.

HaRwood, J.M. and S.R.C. MALIN, Sunspot cycle influence on the geomagnetic field, Geophys. J.R. Astr. Soc., 50, 605-619, 1977.

Malin, S.R.C., Geomagnetic secular variation and its changes, 1942.5 to 1962.5 , Geophys. J.R. Astr. Soc., 17, 415-441, 1969.

MeAD, G.D., Deformation of the geomagnetic field by the solar wind, J. Geophys. Res., 69, 1181-1195, 1964.

Peddie, N. and E.B. Fabiano, A model of the geomagnetic field for 1975, J. Geophys. Res., 81, 2539-2542, 1976.

RIVEN, Yu.R., Spherical analysis of the cyclic variation of the horizontal geomagnetic field component, Geomag. Aeron., 15, 531-532, 1976 (English Trans.).

Sugrura, M., Quiet time magnetospheric field depression at 2.3-3.6 $R_{E}, J$. Geophys. Res., 78, 3182-3185, 1973. 
Sugiura, M. and D.J. Poros, Hourly values of equatorial $D_{\text {st }}$ for the years 1957 to 1970 , NASAGoddard Space Flight Center, X-645-71-278, July 1971.

Vestine, E.H., L. LaPorte, I. LANGe, and W.E. Scott, The geomagnetic field, its description and analyses, Dept. of Terres. Mag. Rpt., No. 580, 390 pp., 1947.

von Neuman, J., R.H. Kent, H.R. Bellinson, and B.I. Hart, The mean square successive difference, Ann. Math. Stat., 12, 153-162, 1941.

World Data Center A for Solar-Terrestrial Physics, Master Station List for Solar-Terrestrial Physics Data at WDC-A for Solar-Terrestrial Physics, Report UAG-38, December 1974.

YukUtake, T., The solar cycle contribution to the secular change in the geomagnetic field, $J$. Geomag. Geoelectr., 17, 287-309, 1965.

Y UKutAKe, T. and J.C. CAIN, Solar cycle variations of the first-degree spherical harmonic components of the goemagnetic field, J. Geomag. Geoelectr., 31, 509-544, 1979.

ZMUdA, A.J. (editor), World Magnetic Survey 1957-1969, IAGA Bulletin No. 28, 1971. 